\title{
Economic growth and control strategies under the environmental pressure: Economic advance-retreat course analysis II
}

\author{
Feng Dai *, Jianping Du, Songtao Wu \\ Department of Management Science, Zhengzhou Information Engineering University No. 850, P. O. Box 1001, \\ Zhengzhou, Henan, 450002, P. R. China
}

(Received October 12 2007, accepted December 6 2007)

\begin{abstract}
ARC (Advance-retreat course) analysis is a theoretical analysis and method on socio-economic development. The main point of ARC is that any economic development behavior will face various pressure coming from environment, that is to say, human beings can actively devise and implement his own economic development strategies, and on the other hand integrative environment formed by all kinds of objective factors can not only passively receive human's choices but aslo human behaviors by the pressure or resistance caused by mankind's behavior. Such dynamic game between human beings and environment is quite different from traditional game problems. Basing on the frame of advance-retreat course (F. Dai, et al, 2007), this paper devises an explanatory model of enterprise development considering environmental pressure according to the basic characteristics of enterprise, develops an analytic model for general advance-retreat course, proves that the solution of the course exists, presents a general method to solve the solution, proposes a series of practical strategies on industry growth control, and particularly draws the important conclusion that faster growing speed will lead to economic growth ending earlier. Finally, the empirical analysis explains ARC to be effective in describing economic growth process, and the controlling strategies for keeping on growth in US economy are given.
\end{abstract}

Keywords: economic growth, advance motivity, environmental pressure, control strategies, ARC (advanceretreat course) analysis

\section{Introduction}

There are many outstanding theoretical studies and achievements in economic growth and development, such as the business cycles ${ }^{[13]}$, the real business cycles ${ }^{[10-12]}$, and the new growth and Endogenous technology ${ }^{[17,18]}$. In recent years, many economists pay more attention the study of the economic growth and development in a variety of ways, such as using biological methods to study the economic growth ${ }^{[1,4,9]}$ and to choose rational economic measures ${ }^{[2,3]}$, the evolution of economic clusters ${ }^{[15,16]}$, the application of game theory to solve economic problems ${ }^{[8,14]}$, and so on. These results are given to study the economic development in the classical, industrial, process and choosing view. However, it is remarkable that, there extensively exists a kind of problems in real world, which is different from those in traditional game theory (both parties are intelligent active behaviors). That is the game between the human who are able to choose the strategies initiatively and environmental pressure with non-active behaviors whose effectiveness often comes out after a period of time. Such as the games between economic development and environmental pollution, enterprise management and market environment, policy making and the implementation environment, knowledge learning and the application environment, energy usage and the resource environment, transportation and the traffic environment, exploitation of natural resources and the ecological environment, and so on. In these problems,

\footnotetext{
*Corresponding author. Tel.: +86-0371-63530975. E-mail address: fengdai@126.com, fengdai@public.zz.ha.cn.
} 
the human as the intelligent and subjective actor will be free to choose and implement the economic strategies, and the environment as non-wisdom objects only passively accept the choices made by human beings. But the environment can act to the human economic behaviors by pressures or resistances. The higher degree the economy develops to and the faster the economy grows, the greater the environmental pressure will be, particularly the pressures brought from the non-reborn resources being gradually dried up and cost of exiguous raw material rising. This means that the process of economic development should have advance or retreat courses, and rise or fall courses. It is similar to the process of boating against the current. The socio-economic behaviors of the human can be various and the environmental pressure is always changing. Such processes known as advance-retreat courses (ARC) ${ }^{[6,7]}$ should not be neglected.

Combining market strategies of developing new products, the authors have discussed discrete ARC models ${ }^{[6]}$, in reference [6]. The basic theory of ARC is studied, so the foundation is laid for developing ARC theory and applying ARC to study economic growth. As a further research, this paper will do following works.

- Designing an explanatory model considering environmental pressure according to the basic characteristics of enterprise development in reality, and discussing the related control problem.

- Perfecting ARC theory further, and studying the existence of the solution of ARC model.

- Giving the way of finding the solution of ARC model under general conditions, and the method to optimally control the time when the benefit reaches maximum.

- Based the US GDP(chained) price indexes, making an empirical analysis about ARC, and making the suggestions about the control strategies that the US economic recession is avoided and growth is kept on.

\section{A model of enterprise production considering environmental pressure and the control strategies for developing}

Here, we are going to develop an ARC model for an enterprise which produces only one type of product. Without loss of generality, the risk-free rate will not be taken into consideration in our model analysis.

\subsection{Notations and problem description}

Assume an enterprise produces only one type of product and raw materials used for production are limited. Here follow some necessary notations.

Assume net motivity $\sigma=u-\bar{u}$ is the key factor that determine the profit of enterprise in assets, that is to say, the enterprise will gain the profit when $u-\bar{u}>0$, thus its benefit increases; the enterprise will not gain the profit when $u-\bar{u}=0$; if $u-\bar{u}<0$, the enterprise will make a loss in profit when $u-\bar{u}<0$, thus its benefit decreases. So, the maximum in enterprise benefit when $\sigma=u-\bar{u}=0$ is a valuable problem, that is, finding $u$ and $\bar{u}$, and having

$$
L\left(T^{*}\right)=\max _{0<t<\infty}\left(\left.L(t)\right|_{u=\bar{u}}\right)
$$

where $\left(T^{*}\right)=u\left(T^{*}\right)-\bar{u}\left(T^{*}\right)=0$ is called equilibrium point of net motivity, and in this case $T^{*}$ and $u\left(T^{*}\right)$ respectively means the equilibrium time and equilibrium strategy in motivity. Following example will illustrate the theoretical significance and practical values of expression (1).

\subsection{An explanatory model of enterprise development considering environmental pressure}

According to the growth model in reference [5], suppose the L(real benefit of enterprise) can be written as

$$
L\left(T^{*}\right)=(t)+h[u(t)-\bar{u}(t)]
$$

where $h$ is growth coefficient of enterprise benefit, $h>0$. Here $h(u-\bar{u})$ can be seen as the profit in assets which is gained based on basic benefit $\mu(t)(t)$ and so $\frac{h[u(t)-\bar{u}(t)]}{\mu(t)}(\mu(t)>0)$ is the growth rate in benefit. 


\begin{tabular}{c|l|l}
\hline$t$ & $:$ & The lasting time of production and development, $0 \leq t<\infty$. \\
\hline$c$ & $:$ & $\begin{array}{l}\text { Total reserve of raw materials. The raw materials are non-renewable when } c \text { is a constant. If } \\
c=c(t)>0 \text { is an increasing function, then raw materials are } \\
\text { renewable. Here, raw materials include other consumed resources. }\end{array}$ \\
\hline$u$ & $:$ & $\begin{array}{l}\text { The motivity for enterprise to produce and develop. } u=u(t)>0 \text { is generally an increasing } \\
\text { function. }\end{array}$ \\
\hline$f_{1}$ & $: \begin{array}{l}\text { Environmental pressure, including varied pressures that affect the production, such as } \\
\text { pressures (problems or difficulties) aroused by capital, techniques, pollution, wastes, } \\
\text { injuries in ecosystem, and so on. } f_{1}=f_{1}[u(t)]>0(t>0) \text { and } f_{1}(u) \text { is an increasing function } \\
\text { on } u .\end{array}$ \\
\hline$f_{2}$ & $: \begin{array}{l}\text { Total quantity of raw materials used by productions. } 0 f_{2}(u)<c \text { (Total quantity of } \\
\text { raw materials is less than that of reserve) and } f_{2}[u(0)]=0 \text { (initial used quantity is zero). }\end{array}$ \\
\hline$f_{3}$ & $: \begin{array}{l}\text { Pressure from production resources. } f_{3}=f_{3}\left(f_{2}, c\right) \text { and } f_{3}\left(f_{2}, c\right) \mid f_{2}=c=+\infty \text { the resource } \\
\text { pressure is infinite when total reserve of raw materials is used up). }\end{array}$ \\
\hline $\bar{u}$ & $: \begin{array}{l}\text { Synthetic pressure of production, including environmental pressure and resource pressure. } \\
\bar{u}=F\left(f_{1}, f_{3}\right)=\bar{f}(u, c) \geq 0 .\end{array}$ \\
\hline$\sigma$ & $: \begin{array}{l}\text { Net motivity for production. If } u>\bar{u}, \sigma=\sigma(u, \bar{u})>0 ; \text { if } u=\bar{u}, \sigma=\sigma(u, \bar{u})=0 ; \\
\text { if } \bar{u}, \sigma=\sigma(u, \bar{u})<0 .\end{array}$ \\
\hline$\mu$ & $: \begin{array}{l}\text { Total assets and resources of enterprise used for production, including fixed asset, current } \\
\text { asset and cost of production. They are the basic benefit for short. } \mu(0)>0 .\end{array}$ \\
\hline$L$ & $: \begin{array}{l}\text { The total of real accumulated net assets of benefit. It is either called the net benefit, and } \\
\text { benefit for short. } L=L(\sigma)=L(\bar{u}) .\end{array}$ \\
\hline
\end{tabular}

When $u=\bar{u}$, net motivity reaches equilibrium, i.e., the motivity and the pressure reaches an equilibrium state.

Let $c(t)=c, \mu(t)=\beta t+\eta, u(t)=a t+b, f_{1}(u)=r u, f_{2}(u)=t, f_{3}(u, c)=\frac{1}{c-f_{2}(u)}=\frac{1}{c-t}$ and $\bar{u}(t)=\frac{r(a t+b)}{c-t}$. Then it follows from expression (2) that

$$
L(t)=\beta t+\eta+h(a t+b)\left[1-\frac{r}{c-t}\right]
$$

${ }^{2}$ where $\beta, \eta, a$ and $b$ are all the given positive constants, $r$ is the environmental pressure index, $0<r<1$, and $f_{2}(u)=c_{1} t\left(0 \leq t<c\right.$ and we might suppose $c_{1} t$ as well) is accumulated quantity of used raw materials at current time. In fact, $t$ can also be seen as the lasting time of production.

Let $u(T)=\bar{u}$ in expression (3), we have $t=T=c-\gamma$. If Let $\frac{d L}{d t}=0$, i.e., $\beta+h\left[a-\frac{r(a c+b)}{(c-t)^{2}}=0\right]$. Since $c-t>0$, we have $\frac{d^{2} L}{d t^{2}}<0$. Thus

$$
T^{*}=c-\sqrt[2]{\frac{h r(a c=b)}{a h+\beta}}
$$

is the time when the benefit reach its maximum. Furthermore, let $T=T^{*}$, then we get pressure index

$$
\gamma=\frac{h r(a c-b)}{a h+\beta}
$$

and

$$
L\left(T^{*}\right)=\frac{\beta(\beta c-h b)}{a h+\beta}
$$

It is shown by expression (4) that the greater $h$ is, the smaller $T^{*}$ is. On the other hand, it is shown by expression (5) that the greater $h$ is, the greater $r$ is. And from expression (6), we see that the greater $h$ is, the small $L T^{*}$ is. Therefore, we draw the following conclusions.

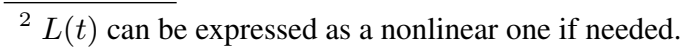


Conclusion 1. From expressions (4), (5) and (6), we can get

(i) The faster the benefit of enterprise production grows, the shorter the time when enterprise benefit reaches maximum will generally be.

(ii) The faster the growing speed of enterprise production is, the greater the pressure faced by the enterprise will be.

(iii) Under the same conditions, the faster the enterprise developing speed is, the smaller the possible maximal benefit of enterprise will be.

So, it is not the better that the growth speed in benefit is too faster for an enterprise to develop continuously.

Let $L\left(T_{1}\right)=\mu\left(T_{1}\right)+h\left[u\left(T_{1}\right)-\left(T_{1}\right)\right]=0$, we have $\beta T_{1}+\eta+h\left(a T_{1}+b\right)\left[1-\frac{\gamma}{c-T_{1}}\right]=0$ and the time when enterprise benefit is equal to zero is

$$
\left(T^{*}\right)=\frac{c \beta-\eta+h[a(c-r)-b]+\sqrt[2]{(c \beta-\eta+h[a(c-r)-b])^{2}+4(\beta+a h)[\eta c+(c-\gamma) h b]}}{2(\beta+a h)}
$$

When $t=T_{1}$, the enterprise can not go on its production since its real benefit is equal to zero.

\subsection{Control method for growth process of enterprise benefit}

In the last subsection, we have given the computing methods of the time when real benefit reaches the maximum and the time when real benefit is equal to zero. In order to effectively control production growth process, it is significant to control the time when real benefit reaches its maximum and the way of benefit growth. In the following, the time when the benefit reaches the maximum and the synthetic weighted method for growth optimal control are given.

\subsubsection{Control and analysis to the time when the benefit reaches its maximum}

Let $T$ be the time when the benefit reaches its maximum. By means of expression (4) and (5) we have

$$
T=c-\frac{h(a c+b)}{a h+\beta}
$$

and the corresponding growth coefficient is

$$
h=\frac{\beta(c-T)}{a T+b}
$$

If $a, b, c$ and $\beta$ in expression (2) are all constants, then it follows from expression (8) that

- When

$$
h>1, T<\frac{\beta c-b}{\beta c+a} .
$$

This implies that the time when the benefit reaches its maximum has a theoretical upper bound.

- When

$$
h=1, T=\frac{\beta c-b}{\beta c+a} .
$$

This implies that the time when the benefit reaches maximum is determinant.

- When

$$
h<1, T>\frac{\beta c-b}{\beta c+a} .
$$

This implies that the time when the benefit reaches maximum has a lower bound.

Thus, the time $\mathrm{T}$ when the net benefit reaches maximum is directly related with the growth coefficient $h$. In detail, when growing speed is faster $(h>1)$, the benefit will reach maximum early, then the increasing time of the benefit is generally shorter; when growing speed is slower $(h<1)$, the benefit will reach maximum later, and then the increasing time of the benefit is generally longer. 


\subsubsection{Optimal control method for growth on weight}

We have shown that it is not the better that the growth speed in benefit is too faster for an enterprise to develop continuously. But if the speed is too slow, the benefit will increase rather slowly, which is also not good for enterprise development. Therefore, taking both growing speed and the benefit into consideration has great realistic significance.

According to expression (6), let us set an auxiliary function $H(h)$ as

$$
H(h)=h^{(1-\omega)} \cdot\left[L\left(T^{*}\right)\right]^{\omega} \cdot \quad(0<\omega<1)
$$

Since

$$
H(h)=e^{(1-\omega) \ln h+\omega \ln L}
$$

and

$$
(1-\omega) \ln h+\omega \ln L=(1-\omega) \ln h+\omega \ln \left[\frac{\beta c-h b}{a h+\beta}\right] \omega \ln \beta,
$$

it follows that

$$
\frac{d H}{d h}=H(h)\left[\frac{1-\omega}{h}-\frac{\omega \beta(a c+b)}{(\beta c-h b)(a h+\beta)}\right]
$$

Let $\frac{d H}{d h}=0$ and have $h>0$, so that we get

$$
h=\frac{\beta}{2 a b(1-\omega)}\left[(1-2 \omega) a c-b+\sqrt[2]{[(1-2 \omega) a c+b]^{2}+4 a b \omega^{2}}\right]
$$

We can deduce from expression (10) that $\frac{1-\omega}{h}=\frac{\omega \beta(a c+b)}{(\beta c-h b)(a h+\beta)}$ Then it follows that

$$
\frac{d^{2} H}{d h^{2}}=-H(h) \frac{1-\omega}{h}\left[\frac{a b h^{2}+c \beta^{2}}{h(\beta c-h b)(a h+\beta)}\right]<0
$$

It can be seen that $h$ determined by expression (10) makes $H(h)=h^{(1-\omega)} \cdot\left[L\left(T^{*}\right)\right]^{\omega}$ in expression (9) reach the maximum, and so $h$ determined by expression (10) is just optimal growth coefficient. Based on weight $\omega$ The enterprise can determine the value of weight $\omega$ according to its needs.

\subsection{An example of controlling enterprise benefit growth}

The enterprise benefit $L(t)=(t)+h[u-\bar{u}]$ is expressed as expression (3). In order to clearly show the significance of the analytical results obtained in the last subsection, a calculation example is presented here. Without loss of generality, assume $c=1$ (reserve in unit), $\mu=\beta t+\eta=0.9 t+0.05$ and $u=a t+b=$ $1.5 t+0.01$.

\subsubsection{Calculation and analysis of synthetic weighted optimal growth results}

Form expression (9) we can get following results about optimal growth based on weight.

(i) Let $\omega=0.7$. We get the optimal growth coefficient $\mathrm{h}$ is equal to 0.44102864 . By expression (5) we have $r=0.4264712938$. Thus, $\bar{u}=\frac{0.4264712938(1.5 t+0.01)}{1-t}$ and at the time $t$ enterprise benefit is

$$
L(t)=0.9 t+0.05+0.3(1.5 t+0.01)\left[1-\frac{0.4264712938}{1-t}\right]
$$

According to expression (4), we get 


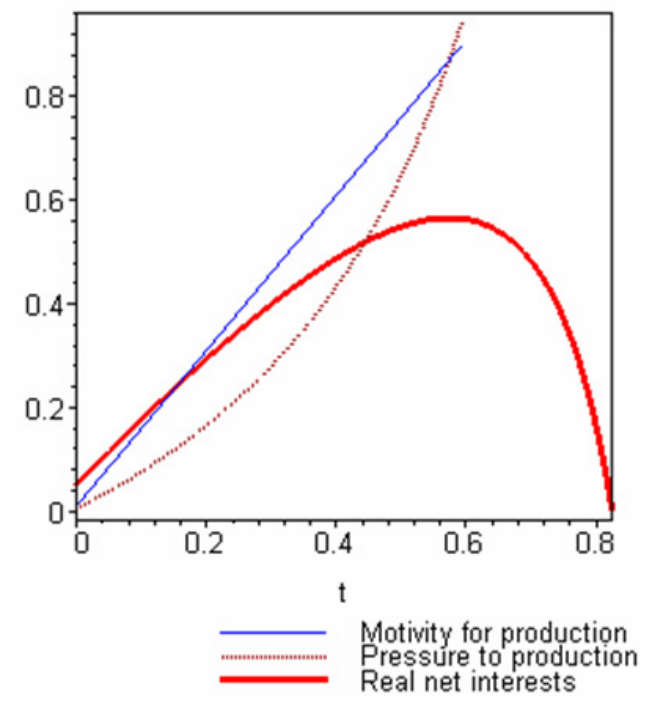

Fig. 1. Enterprise benefit growth when $\omega=0.7$

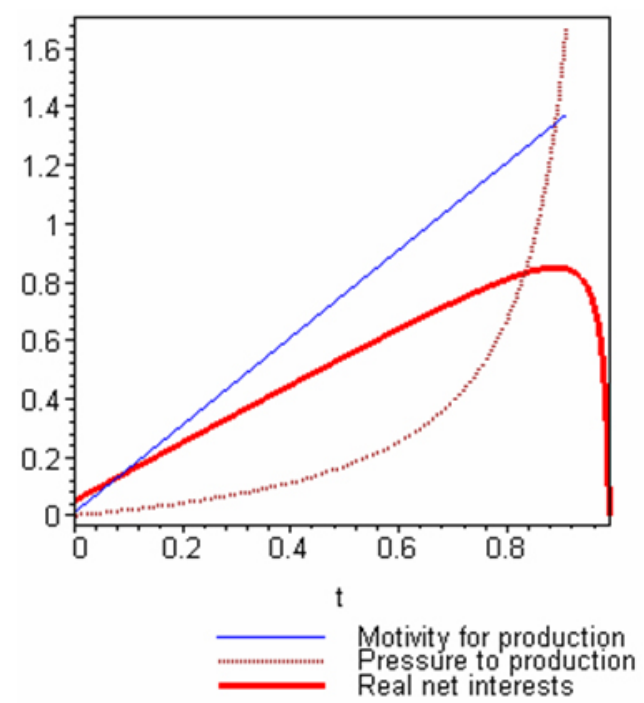

Fig. 2. Enterprise benefit growth when $\omega=0.9$

$$
T^{*}=0.5735287062 \text { and } L_{\max }=L\left(T^{*}\right)=0.5161758356 .
$$

By expression (6) we have $T_{1}=0.8252457322$, here $L\left(T_{1}\right)=0$. See Fig. 1 for some details.

When $\omega=0.7$ it is calculated that $h=0.44102864$. Because the growing speed is relatively fast, the pressure coefficient $r=0.4264712938$ is larger. It results in that both the time when the net benefit attains maximum (equilibrium point of net motivity) and the time when the benefit is equal to zero come early, and the maximal level of benefit is lower, i.e., $L_{\max }=L\left(T^{*}\right)=0.5161758356$.

(ii) Let $\omega=0.9$. We can get the optimal growth coefficient $\mathrm{h}$ is equal to 0.0743724 . Then by (5) we have $r=0.1110190986$. Thus, $\bar{u}=\frac{0.1110190986(1.5 t+0.01)}{1-t}$ and and at the time $t$ enterprise benefit is

$$
L(t)=0.9 t+0.05+0.3(1.5 t+0.01)\left[1-\frac{0.1110190986}{1-t}\right]
$$

According to expression from (4), we get

$$
T^{*}=0.8889809014 \text { and } L_{\max }=L\left(T^{*}\right)=0.8000828112
$$

By (6) we have $T_{1}=0.9882692367$, here $L\left(T_{1}\right)=0$. See Fig. 2 for some details.

When $\omega=0.9$ it is calculated that $h=0.0743724$. Because the growing speed is relatively slow, the pressure coefficient $r=0.1110190986$ is smaller. It results in that both the time when the accumulated net benefit attains maximum and the time when the benefit is equal to zero come early, and the maximal level of benefit is higher, i.e., $L_{\max }=L\left(T^{*}\right)=0.8000828112$.

\subsubsection{Some interesting analysis}

According to the calculation results above, we have the comparing data in Tab. 1.

Table 1. Comparison of key data of enterprise under different weights and growth coefficient

\begin{tabular}{c|c|c}
\hline Benefit weight & Growth coefficient & Pressure coefficient \\
\hline$\omega=0.7$ & $h=0.44102864$ & $r=0.4264712938$ \\
\hline$\omega=0.9$ & $h=0.0743724$ & $r=0.1110190986$ \\
\hline Equilibrium point of motivity & Maximal benefit & Time when the benefit reaches zero \\
\hline$T^{*}=0.5735287062$ & $L\left(T^{*}\right)=0.5161758356$ & $T_{1}=0.8252457322$ \\
\hline$T^{*}=0.8889809014$ & $L\left(T^{*}\right)=0.8000828112$ & $T_{1}=0.9882692367$ \\
\hline
\end{tabular}


Some interesting conclusions are derived form Fig. 1, Fig. 2 and Tab. 1.

Conclusion 2. The following are two basic but important conclusions.

- The net motivity equilibrium point $T^{*}$ only appears for a moment. Generally speaking, the net motivity equilibrium point $T^{*}$ only appears for $a$ moment, and unbalanced net motivity plays the leading role in the whole process.

- It exists that marginal benefit is diminishing. After the appearance of equilibrium point, marginal benefit begins to diminish, which is consistent with the "Marginal Returns Diminishing" phenomena in economic field.

Conclusion 3. Comparing the track of the curves in Fig. 1 and Fig. 2 and analyzing the data in Tab. 1, following conclusions can be obtained.

- If the growth coefficient of enterprise production is larger (i.e., the growing speed of the production is faster), then the environmental pressure to enterprise production will be larger and faster in increase. On the contrary, if the growing speed of enterprise production is slower, then the environmental pressure to production will be smaller and slower in increase.

- If the growing speed of enterprise production is faster, then the equilibrium point of net benefit will appear more early, i.e., the enterprise benefit will reaches its maximum more early. On the contrary, if the growing speed of enterprise production is slower, the enterprise benefit will reaches its maximum more lately, i.e., the increasing process in benefit will keep on for a longer time.

- The faster growing speed of the production will result in that the enterprise gain smaller benefit in the end. On the contrary, slower growing speed of the production will result in that the enterprise gain more benefit in the end.

- The growing speed is faster unduly in order to gain more benefit may only result in smaller benefit be gained.

The explanatory conclusions can be as follows through the analysis above.

Conclusion 4. About enterprise production and development, the following conclusions are worthy to be paid attention to.

- Any raw material will not be used out, since if exploring and using raw materials exceed a certain degree, the cost (or price of raw materials) will increase so much that the enterprise is difficult to receive. In fact, after the time $T^{*}$, such production can not make the real benefit increase any more.

- As for an enterprise, there exists an critical time and an ending time to produce every kind of products. Between the critical time and an ending time, the enterprise benefit returned from producing the product will reduce, and should take into consideration of decreasing or ending the production. The suitable period of time is between $T^{*}$ and $T_{1}$.

- In the process of production and sale, the enterprise maximal benefit appears only in a moment. Therefore, catching the opportunity and adopting proper policies is important to keep on developing an enterprise.

- When a product is going to the ending time of its production, some new kind of product is needed to replace the old one, and so the enterprise will begin a new process of production. At this time, the enterprise needs to make an investment to research or buy the new techniques for the new kind of product, or to turn into some of other fields in economy and some new energies may be used.

It is worth to say, based on the simple model above, that the enterprise can actively choose raw materials and strategies for production, and though the production environments (including total reserve of raw materials, market environment) can only passively receive the choices made by the enterprise, they can affect the enterprise benefit in the way of pressure. In the influential process, the enterprise is advancing when its benefit gradually increases, and it is retreating when its benefit decreases.

\subsubsection{Control and analysis to the time when the benefit reaches maximum}

According to expression (4) and (5), we get the time $T=c-\frac{h(a c+b)}{a h+\beta}$ at which the benefit reaches maximum, and then 


$$
h=\frac{\beta(c-T)}{b+a T}
$$

The corresponding environmental pressure index is $r=\frac{h(a c+b)}{a h+\beta}$. So the enterprise can control its growth coefficient $h$ freely by the expression (11) in order to determine the expected time that the benefit reaches maximum.

Here we gives two examples of controlling the time at which benefit reaches maximum, the related computing data are in Tab. 2, and the process of benefit changing is shown in Fig. 3.

Table 2. Computing data for controlling time when the maximal benefit appears

\begin{tabular}{c|c|c}
\hline Time when maximal benefit appears & Growth coefficient & Pressure coefficient \\
\hline$T=0.4$ & $h=0.8852459016$ & $r=0.6$ \\
\hline$T=0.9$ & $h=0.06617647059$ & $r=0.1$ \\
\hline Value of maximal benefit & \multicolumn{2}{|c}{ Time when benefit is equal to zero } \\
\hline$L_{\max }=0.41$ & \multicolumn{2}{|c}{$T_{1}=0.6527828910$} \\
\hline$L_{\max }=0.86$ & \multicolumn{2}{|c}{$T_{1}=0.9904863306$} \\
\hline
\end{tabular}

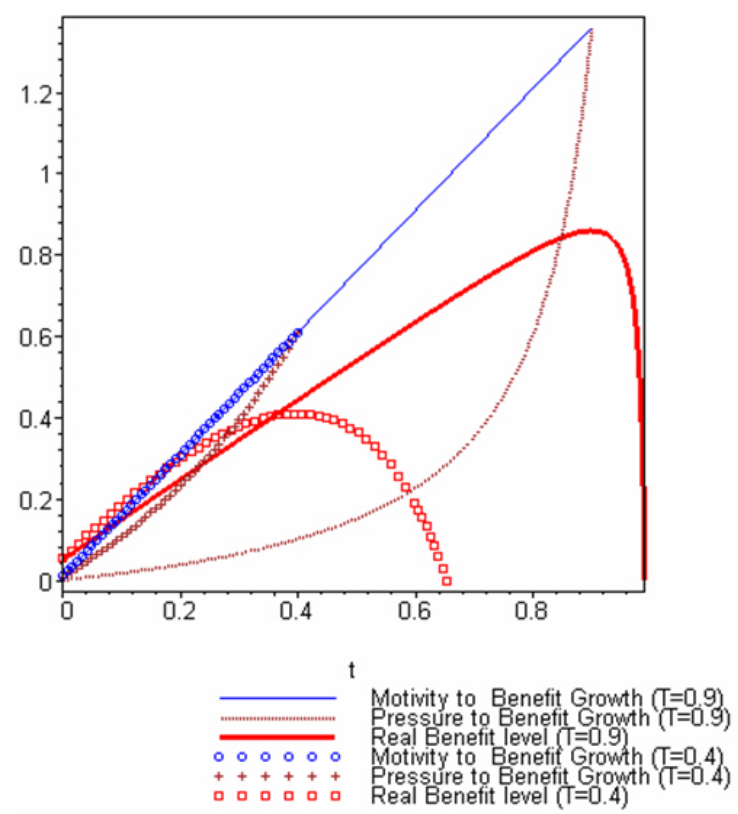

Fig. 3. Comparison of benefit on different period of time

By comparing the growth processes in which benefit reaches maximum at the different time $(T=0.4$ and $T=0.9$ ), we see, under the same motivity for production, that the benefit will reaches its maximum earlier if $T=0.4$, at same time, the growth coefficient is larger $(h=0.8852459016)$ and the pressure index is also larger $(r=0.6)$, and the maximal benefit is smaller $\left(L_{\max }=0.41\right)$. The benefit will reaches its maximum later if $T=0.9$, and the growth coefficient is smaller $(h=0.06617647059)$ and the pressure index is also smaller (0.1), and the maximal benefit is larger $\left(L_{\max }=0.86\right)$.

Conclusion 5. From Tab. 2 and Fig. 3, we can analyze to get following conclusions.

- If the maximal benefit is controlled to be reached at $T=0.4$, the earlier time, then we have $h=$ 0.885245901 which is higher. In such case, the benefit increases faster at the beginning, but the final maximal benefit is smaller, that is $L_{\max }=0.41$. The lasting ability of such production growth is weaker.

- If the maximal benefit is controlled to be reached at $T=0.9$, then we have $h=0.06617647059$. In such case, although the benefit increases in a slower way at the beginning, the final maximal benefit is larger, that is $L_{\max }=0.86$. The lasting ability of such production growth is stronger. 
- If the enterprise expects a high level of benefit and a longer time of growth, it should effectively control its growth speed. In fact, the reasonably slower speed in growth will result in greater maximal benefit.

According to Conclusion 5, there are two different production and development strategies for the enterprise to choose as following.

Strategy 2.1. Growing in a faster speed, the enterprise can realize a higher level of benefit in a short time at the beginning. Its growth in benefit, however, cannot keep on a longer time, and so the increasing process of the benefit will be shorter, and the final maximal benefit will be lower.

Strategy 2.2. Growing in a slower speed, the enterprise can finally realize a higher level of benefit, however, the time, at which benefit reaches maximum, will be later. In this case, the growth will keep on a longer time, and the level of maximal benefit will finally be higher.

\section{The basic theory on advance-retreat course (arc) analysis}

The growth and development process of anything includes two basic stages, advance (rise) stage and retreat (fall) stage, and the interactions between motivity and resistance must exist in the process. Based on this view, we call the process an advance-retreat course.

\subsection{The concept system of advance-retreat analysis}

Based on the abovementioned various cases, the basic elements, basic characteristics and types of countermeasures of advance-retreat analysis can be summed up. Then the basic concept system of advance- retreat course can be synthesized.

\subsubsection{The basic elements of advance-retreat course}

The advance-retreat course consists of two pair basic elements, the initiative actors (subject for short) and the integrative environment (object for short) in advance course, and the forward motivity for the subject and the pressure from the object ${ }^{[6]}$.

Definition 1. (subject and object) The subject is the actor who takes the initiative to choose strategies and put the strategies into practice. The dominate behavior of the subject is moving in the direction of progressing and developing. The object refers to the objective things which hinder the subject from progress. The dominate behavior of the object is to passively resist on the subject's advancing.

The various legal requirements of the subject are called the benefit of the subject (benefit for short), including requirements for capital, assets, resources, services, and wishes, and, etc. The net benefit is the remaining benefit after the loss is subtracted, and is also called benefit for short if there is no confusion.

Definition 2. (motivity) The motivity for the subject is the power pushing the subject to advance, which is also called as the power for the subject. The motivity includes endogenous motivity and exogenous motivity. The endogenous motivity is from the subject himelf and his internal environment, and the exogenous motivity is from the related environment elements outside the subject.

Definition 3. (pressure) The resistance from the object is the force that hinders the subject from advancing, and it is also called as environmental pressure or resistance, including endogenous pressure and exogenous pressure. The pressure caused by the changes in the internal environment which is composed of the subject's own factors, is called as endogenous pressure, and the pressure as a result of changes in the external environment which is composed of factors outside the subject, is called as exogenous pressure.

The net forward motivity is the remaining part after the pressure is subtracted from the motivity. That is the net motivity for short.

Assumption 1. (Basic assumption) All the things in socio-economic field have their affections to others, including the subject and object. Some related affections will make subject's benefit increase or decrease, and can be distinguished as motivity or pressure. Their affection can be evaluated by fluctuation variance of state level of underlying things. 
Definition 4. (advance, indirect advance and retreat) The movement along with the increasing of the net benefit for the subject is called as advance. The movement with the net benefit keeping unchanged is called as indirect advance, and the movement along with the decreasing of net benefit is called as retreat.

According to Assumption 2, we know that the direction of the motivity is consisting with the advance direction, while the direction of the pressure is opposite to the advance direction.

\subsubsection{The basic strategies for subject in advance-retreat course}

In order to have an effective description on strategies in the advance-retreat course, the following marks are given out.

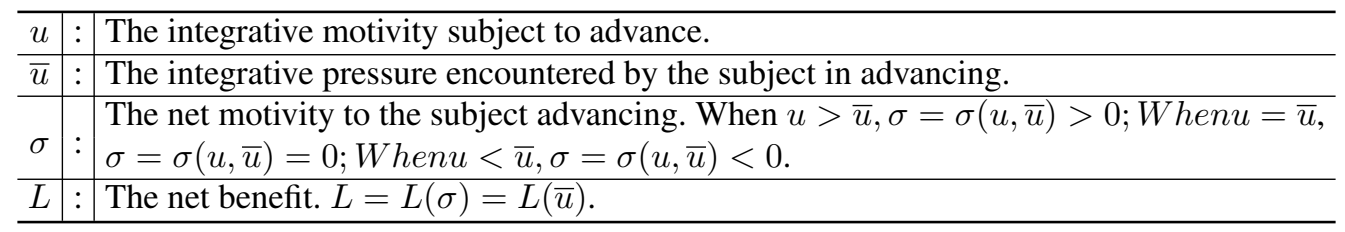

Normally, there are three countermeasures for the subject in the advace-retreat course.

- When $u-\bar{u}>0$, the net forward motivity $\sigma=\sigma(u, \bar{u})>0$, then the subject can choose advance, and the net benefit $L(\bar{u})$ will increase.

- When $u-\bar{u}=0$, the net forward motivity $\sigma=\sigma(u, \bar{u})=0$, then the subject generally chooses to stay here, and net benefit $L(\bar{u})$ will keep no change (here the cost for staying is ignored). The subject may also adopt measures to enhance motivity and to continue to advance, or choose temporary retreat under specific circumstances.

- When $u-\bar{u}<0$, the net forward motivity $\sigma=\sigma(u, \bar{u})<0$, then it is feasible for the subject to choose retreat, and net benefit $L(\bar{u})$ will decrease. The subject may also adopt measures to enhance motivity and to continue to advance.

\subsection{Advance-retreat course and the existence of its solution}

In the following discussion $\zeta$-accumulated utility ${ }^{[6]}$ is always used to measure the subject's benefit, that is

$$
L[\sigma(t)]=H\left(\int_{0}^{t} \zeta(\tau) \sigma(\tau) d(\tau)\right)
$$

where

$$
\zeta(t)>0 \quad \text { and } \quad \zeta(t) \in C^{(0)}
$$

$C^{(i)}$ Pis a set of all $i$-order continuous and differentiable functions, $i=0,1, \cdots$. Without confusion, $\zeta$ accumulated utility $L[\sigma(t)]$ is also called benefit for short, where $\sigma(t)$ is net motivity.

Definition 5. (course) Course is the shortened form of the advance-retreat course, or ARC for short. It can be divided as continuous course and discrete course, and the definitions of them are

(i) The net motivity $\sigma(t)$ and the benefit $L[\sigma(t)]$ are continuous, $\sigma(0)>0$ and $L[\sigma(0)]>0$. If there is a moment $\bar{t}$ with $\bar{t}>0$ or $\bar{t}=+\infty$, and when

$$
t \in[0, \bar{t}), L[\sigma(t)]>0 \quad \text { and } \quad L[\sigma(\bar{t})]=0, L[\sigma(t)](t \in[0, \bar{t}])
$$

is called as a continuous course. 
(ii) For the net motivity $\sigma_{i}(i=0,1, \cdots, n)$ and the benefit $L\left(\sigma_{i}\right), \sigma_{0}>0$ and $L\left[\sigma_{0}\right]>0$. If there is $n_{0} \in[0, n]$, at some moment $L\left[\sigma_{i}\right]>0\left(i=0,1, \cdots, n_{0}-1\right)$ and $L\left[\sigma_{n 0}\right]=0, L\left[\sigma_{i}\right], \in\left[0, n_{0}\right]$ is called as a discrete course.

Based on the analysis above, the course model can be generally represented as

$$
L=L(\sigma)=H(u, \bar{u})
$$

where the notations are the same as in section 3.1.2.

Definition 6. (solution of course) For a continuous course $L\left[\sigma_{i}\right](t \in[0, \bar{t}], \bar{t}$ is finite or $\bar{t}=+\infty)$, if there exists an $T \in(0, \bar{t})$ for which

$$
L[\sigma(T)]=\max _{0<t<\bar{t}} L[\sigma(T)] \quad \text { and } \quad \sigma(T)=0
$$

then $L[\sigma(T)]$ is defined as a solution of the continuous course $L[\sigma(t)](t \in[0, \bar{t}])$. For a discrete course $L\left(\sigma_{i}\right)(i=0,1, \cdots, n)$, if there exists an $T \in(0, n)$, for which

$$
L\left(\sigma_{T}\right)=\max _{0<i<n} L\left(\sigma_{i}\right) \quad \text { and } \quad \sigma_{T}=0,
$$

then $L\left(\sigma_{T}\right)$ is defined as a solution of the discrete course $L\left(\sigma_{i}\right)(i=0,1, \cdots, n)$.

Definition 7. (equilibrium point) If $t>0$ and $\sigma(t)=\sigma[u(t), \bar{t}(t)]=0$ then $t$ is called equilibrium point of net motivity.

According to Definition 7, the solution of a course can be thought as the maximal accumulated net benefit at equilibrium point of net motivity.

Theorem 1. (existence of course solution).

(i) For a continuous course,

$$
L\left[\sigma_{i}\right]\left(t \in[0, \bar{t}], L[\sigma(t)] \in C^{(2)} \quad \text { and } \quad \sigma(t) \in C^{(1)},\right.
$$

if there exists an $T \in(0, \bar{t})$ for which

$$
\sigma(T)=0 \quad \text { and } \quad \frac{d^{2} L(T)}{d t^{2}}<0,
$$

then the solution of thecourse $L[\sigma(t)]$ exists.

where $C^{(i)}$ is a set of all $i$-order continuous and differentiable functions.

(ii) If a discrete course $L\left[\sigma_{i}\right]$ and $\sigma_{i}(i=i=0,1, \cdots, n)$ have an upper bound, that is, there exists an $T \in[0, n]$ for which

$$
L\left(\sigma_{T}\right)=\max _{0<i<n} L\left(\sigma_{i}\right),
$$

then $L\left(U_{T}\right)$ is the solution of $L\left(\sigma_{i}\right)(i=0,1, \cdots, n)$.

Proof. (i) According to Definition 5, we have

$$
L[\sigma(t)]=H\left(\int_{0}^{t} \zeta(\tau) \sigma(\tau) d(\tau)\right) \in C^{(2)},
$$

Then it follows from the condition of the theorem that

$$
\left.\frac{d L}{d T}\right|_{t=T}=H^{\prime} \zeta(T) \sigma(T)=0 \quad \text { and }\left.\quad \frac{d^{2} L}{d t^{2}}\right|_{t=T}=\left.H^{\prime} \zeta(T) \sigma(T)\right|_{t=T}<0 .
$$

Therefore, 


$$
L[\sigma(T)]=\max _{0<t<\bar{t}} L[\sigma(T)]
$$

is the solution of the course.

(ii) According to Definition 5,

$$
L\left(\sigma_{i}\right)=H\left[\sum_{s=1}^{i} \zeta(s) \sigma(s)\right]
$$

and $\sigma_{i}$ has an upper bound. Since $H(t)$ is an increasing function and $\zeta(i)>0$, it follows that there exists an $i_{0}$ such that

$$
L\left(\sigma_{i_{0}}\right)=H\left[\sum_{s=1}^{i_{0}} \zeta(s) \sigma(s)\right]=\max _{1 \leq i<n} H\left[\sum_{s=1}^{i_{0}} \zeta(s) \sigma(s)\right] .
$$

If $\sigma_{i_{0}}=0$, then $L\left(\sigma_{i_{0}}\right)$ is the solution of the course $L\left(\sigma_{i}\right)(i=0,1, \cdots, n)$ If $\sigma_{i_{0}} 0$, then $\sigma_{i_{0}}>0$

$$
\text { (otherwise, } \quad H\left[\sum_{s=1}^{i_{0}} \zeta(s) \sigma(s)\right]<H\left[\sum_{s=1}^{i_{0}-1} \zeta(s) \sigma(s)\right] \text {, a contradiction), }
$$

and so that $\sigma_{i_{0}+1} \leq 0$

$$
\text { (otherwise, } \quad H\left[\sum_{s=1}^{i_{0}} \zeta(s) \sigma(s)\right]<H\left[\sum_{s=1}^{i_{0}+1} \zeta(s) \sigma(s)\right] \text {, a contradiction). }
$$

If $\sigma_{i_{0}+1}=0$, then $L\left(\sigma_{i_{0}+1}\right)$ is the solution of the course $L\left(\sigma_{i}\right)(i=0,1, \cdots, n)$. Otherwise, let

$$
\sigma_{i_{0}+k+1}=\sigma_{i_{0}+k}\left(k=1, \cdots, n-i_{0}\right) \quad \text { and } \quad \sigma_{i_{0}+1}=0 .
$$

Then $L\left(\sigma_{i_{0}+1}\right)=L\left(\sigma_{i_{0}}\right)$ is the solution of the course $L\left(\sigma_{i}\right)(i=0,1, \cdots, n+1)$, so it is also the solution of the course $L\left(\sigma_{i}\right)(i=i=0,1, \cdots, n)$.

\section{The advance-retreat course model considering environmental pressure}

A description model of the advance-retreat model is got by expression (12). In the following we will give the economic ARC model with which the environmental pressure is considered, and discuss the control strategies of the time when the subject's benefit reaches its maximum based on the course model.

\subsection{General model of the arc and its solution}

\subsubsection{The notations and basic arc model}

First we give some necessary notations.

Because the subject needs some initial capital (initial basic benefit) to start a course, the course model considering basic benefit and environmental pressure can be expressed as

$$
L=L\left(\mu_{L}, \sigma_{L}\right)=H(\mu, \bar{\mu}, u, \bar{u})
$$

Based on model (13) and take the dynamic characteristic of the course into consideration, we have $\mu_{L}=$ $\mu_{L}(t), \sigma_{L}=\sigma_{L}[u(t), \bar{u}(t)], L=L\left[\mu_{L}(t), \sigma_{L}(t)\right], t \leq 0$. If $\mu_{L}(t), \sigma_{L}[u(t), \bar{u}(t)]$ and $L\left[\mu_{L}(t), \sigma_{L}(t)\right]$ are differentiable, the solution $L(T)$ of the course is determined by the following equations:

$$
\left\{\begin{array}{l}
\sigma_{L}(T)=0 \\
L^{\prime}(T)=H_{1}^{\prime} \mu_{L(T)}^{\prime}+H_{2}^{\prime} \sigma_{L(T)}^{\prime}=0
\end{array}\right.
$$




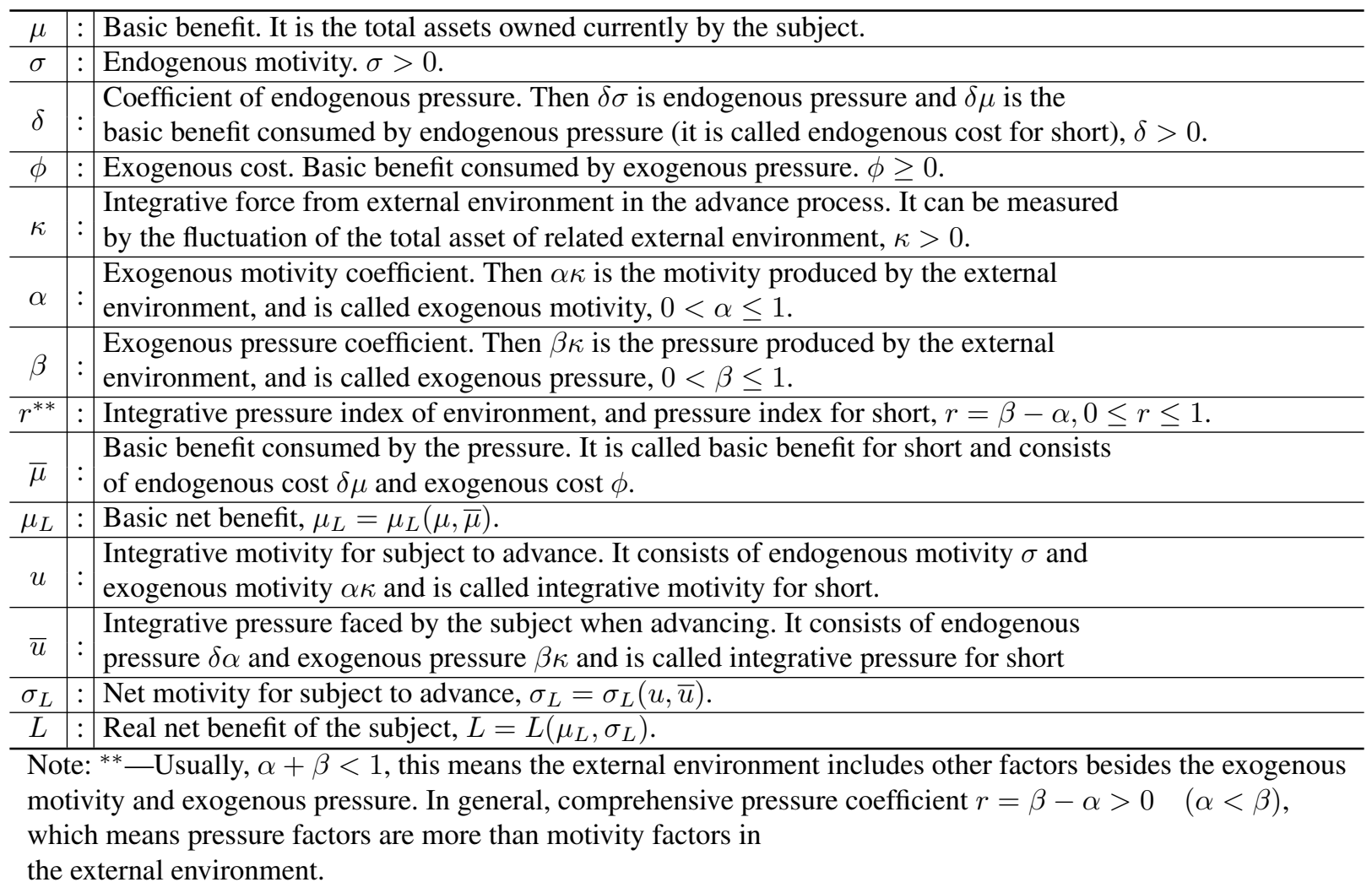

Theorem 2. If the course is a single-peak function and its solution exists, the marginal net benefit will decrease after the time corresponding to the solution.

Proof. If $L(T)$ is the solution of the course

$$
L(T)\left(0 \leq t \leq T_{1}\right), L(T)=\max _{0 \leq t \leq T_{1}} L(T),
$$

where $L\left(T_{1}\right)=0 . T$ is the only time that satisfies (14). Since $L(t)$ is a single-peak function, it follows that $L^{\prime}(t)<0$ if $t<T$, that is, the marginal net benefit decreases.

\subsubsection{Linear course model and its solution}

In particular, if basic net benefit can be linearly expressed as $\mu_{L}(t)=(1-\delta) \mu(t)-\phi(t)$, integrative motivity can be linearly expressed as $\mu=\sigma(t)+\alpha \kappa(t)$, integrative pressure can be linearly expressed as $\bar{u}=\delta \sigma(t)+\beta \kappa(t)$, and based on the result ${ }^{[5]}$, ARC model (13) can be expressed as

$$
L(T)=\mu_{L}(t)+h \sigma_{L}(t)=(1-\delta) \mu(t)-\phi(t)+h[(1-\delta) \sigma(t)-r \kappa(t)]
$$

${ }^{3}$ where $h>0$ is called benefit growth coefficient.

If $\mu_{L}(t)>0$, model (15) can be expressed as $L(t)=\mu_{L}(t)(1+g)$, where $g=h \frac{\sigma_{L}(t)}{\mu_{L}(t)}$ is the real benefit growth rate. If $\mu_{L}(t)$ and $\sigma_{L}(t)$ in the model (15) are differentiable, the $\mathrm{T}$ in its solution $L(T)$ can be determined by the following equations:

$$
\left\{\begin{array}{l}
\sigma_{L}(T)=0 \\
L^{\prime}(T)=\mu_{L(T)}^{\prime}+h \sigma_{L(T)}^{\prime}=0
\end{array}\right.
$$

\subsection{The control strategies for subject}

According to the model (15), we can make following analysis to control a course.

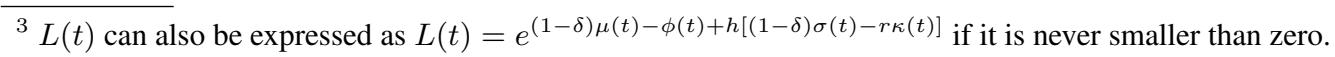




\subsubsection{Controlling the time at which benefit reaches maximum}

If the maximal benefit is expected to appear at the time $T$, let $\sigma_{L}(T)=0$ in model (15), i.e., based on expression (16), it follows that

$$
r^{0}=\frac{(1-\delta) \sigma(T)}{\kappa(T)}
$$

And by the equations (16), if $\mu_{L}^{\prime}(T)>0$, and we get

$$
h^{0}=\frac{\mu_{L}^{\prime}(T)}{\sigma_{L}^{\prime}(T)}
$$

where, $h^{0}>0$ because $\sigma_{L}^{\prime}(T)<0$

Thus, $L(T)$ is the solution of the course $L(t)=\mu_{L}(t)+h^{0} \sigma_{L}(t)$, where $\sigma_{L}(T)=(1-\delta) \sigma(t)-r^{0} \kappa(t)$ In addition, if

$$
\mu_{L}(t) \neq 0, \quad L(t)=\mu_{L}(t)+h^{0} \sigma_{L}(t)
$$

can be expressed as $L(t)=\mu_{L}(t)[1+g(t)]$, where benefit growth rate $g(t)=h^{0} \frac{\sigma_{L}(t)}{\mu_{L}(t)}$. If $\mu_{L}(t)$ and $\sigma_{L}(t)$ are continuous on the interval $[0, T]$, then the average growth rate of subject benefit is

$$
\bar{g}=\frac{h^{0} \int_{0}^{T} \frac{\sigma_{L}(t)}{\mu_{L}(t)} d(t)}{T}
$$

\subsubsection{Weighted optimal control to the benefit growth coefficient}

If $L(T)$ is the solution of the course $L(t)$, then we can build an auxiliary function

$$
A(h)=h^{(1-\omega)} \cdot[L(T)]^{\omega}
$$

where $\omega(0<\omega<1)$ is the benefit weight and $(1-\omega)$ denotes the weight of benefit growth coefficient.

According to equations (16), we can solve the time $T=T(h)$ when benefit attains maximum, and $\sigma_{L}(T)=0$ at the same time. Then combining with model (15), the auxiliary function (20) is converted into

$$
A(h)=h^{(1-\omega)} \cdot[L(T)]^{\omega} .
$$

Denote $\mu_{L}=\mu_{L}(T), \mu_{L}^{\prime}=\left[\mu_{L}(T)\right]_{t}^{\prime}$ and $T^{\prime}=T_{h}^{\prime}(h)$. Let

$$
\frac{d A}{d h}=A(h)\left[\frac{1-\omega}{h}+\frac{\omega \mu_{L}^{\prime} T^{\prime}}{\mu_{L}}\right]=0 .
$$

Then it can be calculated that $h=h(\omega)$. Since $A(h)>0$, we have $h=-\frac{(1-\omega) \mu_{L}}{\omega \mu_{L}^{\prime} T^{\prime}}$, and it follows that

$$
\left.\frac{d^{2} A}{d h^{2}}\right|_{h=h(\omega)}=-\frac{\omega A(h)}{(1-\omega)\left(\mu_{L}\right)^{2}}\left[\left(\mu_{L}^{\prime} T^{\prime}\right)^{2}+(\omega-1)\left(\mu_{L}^{\prime} T^{\prime}\right)^{\prime} \mu_{L}\right]
$$

It can be seen that whether

$$
\left(\mu_{L}^{\prime} T^{\prime}\right)^{\prime} \mu_{L}=0 \operatorname{or}\left(\mu_{L}^{\prime} T^{\prime}\right)^{\prime} \mu_{L} \neq 0 \quad \text { and } \quad 1>\omega>1-\frac{\left.\mu_{L}^{\prime} T^{\prime}\right)^{2}}{\left(\mu_{L}^{\prime} T^{\prime}\right)^{\prime} \mu_{L}}=1-\frac{d \ln \left(\mu_{L}\right)}{d \ln \left(\mu_{L}^{\prime} T^{\prime}\right)}, \frac{d^{2} A}{d h^{2}}<0,
$$

so $A(h)=h^{(1-\omega)} \cdot[L(T)]^{\omega}$ reaches maximum. 


\section{An empirical analysis for controlling us economic growth}

In the following, an empirical analysis for controlling US economic growth will be given to check the validity of ARC model and the control strategies.

\subsection{An empirical model and data}

At first, an empirical model and data to be used in the empirical analysis are given.

\subsubsection{An empirical model}

The empirical analysis uses model (15), and we give the following assumptions.

Assumption 2. If basic benefit and motivity during the advancing process is relevant to the time, then

(i) Because the basic benefit should increase naturally according to the risk-free rates in the case of no pressure, so we suppose that $\mu(t)=\mu_{0} e^{\lambda t}$, where $\mu_{0}$ is initial basic benefit and $\lambda$ is natural growth rate (i.e., risk-free rate) of benefit, $\lambda>0$. As the natural growth of the basic benefit, endogenous motivity also grow in the same rate, i.e., $\sigma(t)=\sigma_{0} e^{\lambda t}$, where 0 is the initial endogenous motivity.

(ii) Because the subject will continuously expect to acquire more benefits along with technique progress and efficiency increase, so its motivity will grow continuously also. Taking the natural growth factors into consideration, we suppose the endogenous motivity increases in the way of $\sigma(t)=\sigma_{0} q(t) e^{\lambda t} e^{\lambda t}$, where $q(t)$ is the integrative innovational factor and increase by degree, $q(0)=1$.

Assumption 3. If the exogenous cost and the environmental action will change as the endogenous motivity changes and time elapses when the subject advances, then

(i) Because the integrative action of environment will also grow to subject as endogenous motivity grows, we suppose that the integrative action has a non-linearly expression form, i.e., $\kappa(t)=\kappa_{0} q^{\theta}(t) e^{\psi t}$, where $q^{\theta}(t)$ is applied to measure the part of environmental action caused by technique power, $e^{\psi t}$ measure the environmental action caused by the natural growth of the endogenous motivity, $\theta>1$ and $\psi>\lambda$ means that the endogenous motivity increasing will result in the greater growth of environmental action.

(ii) Because the pressure will increase as environmental action increases, so we suppose that the exogenous cost increases as environmental action increases naturally, i.e., $\phi(t)=\phi_{0} e^{\psi t} S$, where $\phi_{0}$ is the initial exogenous basic cost.

According to the two assumptions above, the integrative motivity can be linearly expressed as $u(t)=$ $\sigma_{0} q(t) e^{\lambda t}+\alpha \kappa_{0} q^{\theta}(t) e^{\psi t}$, integrative pressure can be $\bar{u}=\delta \sigma_{0} q(t) e^{\lambda t}+\beta \kappa_{0} q^{\theta}(t) e^{\psi t}$, and integrative basic cost can be $\delta \mu_{0} e^{\lambda t}+e^{\psi t}$. Especially, if innovational factor $q(t)$ is given as $q(t)=(1+s t)$ where $s(>0)$ is the innovational coefficient, then real benefit is

$$
L(t)=\mu_{L}(t)+h \sigma_{L}(t)
$$

where $\mu_{L}(t)=(1-\delta) \mu_{0} e^{\lambda t}-\phi_{0} e^{\psi t}$ is the basic net benefit,

$$
\sigma_{L}(t)=\sigma_{L}(t)=(1-\delta) \sigma_{0}(1+s t) e^{\lambda t}-r \kappa_{0}(1+s t)^{\theta} e^{\psi t}
$$

is the net motivity and $r=\beta-\alpha>0$ is the pressure index. In fact, both motivity and pressure are always existing simultaneously, then the initial data that can be directly obtained are the initial benefit $\mu_{L}(0)=$ $(1-\delta) \mu_{0}-\phi_{0}$ and the initial motivity $\sigma_{L}(0)=(1-\delta) \sigma_{0}-r \kappa_{0}$. Thus we have

$$
\mu_{0}=\frac{\mu_{L}(0)+\phi_{0}}{1-\delta} \quad \text { and } \quad \frac{\sigma_{0}=\sigma_{L}(0)+r \kappa_{0}}{1-\delta}
$$




\subsubsection{Controlling the time on which benefit attains maximum}

If the maximal benefit is expected to obtain at time $T$, then by expression (16), (17) and (22) we get

$$
r=\frac{\sigma_{L}(0)}{\kappa_{0}\left[e^{(\psi-\lambda) T}(1+s T)^{\theta_{1}}-1\right]}
$$

and by the expression (18) and (22), the formula to calculate the benefit growth coefficient

$$
h=\frac{\lambda\left(\mu_{L}(0)+\phi_{0}\right)-\frac{\psi \phi_{0}}{(1-s T)^{\theta-1}}\left[1+\frac{\sigma_{L}(0)}{r \kappa_{0}}\right]}{\left[\sigma_{L}(0)+r \kappa_{0}\right][(\theta-1) s+(\psi-\lambda)(1+s T)]}
$$

where

$$
\lambda\left(\mu_{L}(0)+\phi_{0}\right)-\frac{\psi \phi_{0}}{(1-s T)^{\theta-1}}\left[1+\frac{\sigma_{L}(0)}{r \kappa_{0}}\right]>0
$$

that is

$$
1>\frac{\lambda}{\psi}>\frac{\phi_{0}}{\left(\mu_{L}(0)+\phi_{0}\right)(1-s T)^{\theta-1}}\left[1+\frac{\sigma_{L}(0)}{r \kappa_{0}}\right]
$$

The inequality (25) reflects the conditional relation between natural growth rate of benefit and growth rate of environmental factors, and so that the peak of the course can be controlled by adjusting them.

From expression (22) and (23), the solution of the course (21) after controlling is as follows

$$
L(T)\left[\mu_{L}(0)+\phi_{0}\right] e^{\lambda T}-\phi_{0} e^{\psi T}+h\left[\left(\sigma_{0}+r \kappa_{0}\right)(1+s T) e^{\lambda T}-r \kappa_{0}(1+s T)^{\theta} e^{\psi T}\right]
$$

From expression (26), the loss of real benefit consume, caused by exogenous pressure, can be measured

$$
W(T)=h r \kappa_{0}(1+s T)^{\theta} e^{\psi T}
$$

\subsubsection{Data and initial values}

We use GDP(chained) price index data of America during 1940 and $2006^{4}$ in the following empirical analysis. $G(t)$ denotes the th year of American GDP price index, $t=1940,1941, \cdots, 2006$.

Since $G(1940)=0.0978, G(1941)=0.1014$, the initial values can be determined as $\mu_{L}(0)=$ $G(1941)=0.1014, \quad \sigma_{L}(0)=G(1941)-G(1940)=0.0036^{5}$. Assume $\delta=0.3, \phi_{0}=0.01, \kappa_{0}=0.02, s=$ $0.2, \theta=2.0$.

In the following, the fitting errors are measured by the following formulas

$$
\epsilon_{1941}=\sqrt[2]{\frac{\sum_{t=1941}^{2006}[L(t)-G(t)]^{2}}{2006-1941}}, \quad \epsilon_{1985}=\sqrt[2]{\frac{\sum_{t=1985}^{2006}[L(t)-G(t)]^{2}}{2006-1985}}
$$

where $L(t)$ is the benefit estimated.

\subsection{Controlling and analyzing for us economic arc}

Now, many economists consider that US economy will depress soon. By the ARC method, here we will give the controlling strategies for US economy to grow till $T=2015$ or $T=2020$.

\footnotetext{
${ }^{4}$ Data is cited from http://www.whitehouse.gov. Here we measure economic benefit by using of GDP price index. Because economic production is made on the basis of last year, so GDP price index can be seen as a kind of accumulated result of previous GDP price indexes.

${ }^{5} \sigma_{L}(0)$ - The initial fluctuating range of GDP, $\sigma(0)=|\mu(1)-\mu(0)|$. Since the interval unit of GDP data is one year, GDP data can reflect stably the economic status, so the $\sigma(0)=|\mu(1)-\mu(0)|$ can describe nicely economic fluctuation.
} 


\subsubsection{Arc controlling and strategies analysis for us economy to grow till $t=2015$}

If US economic benefit is controlled to reach its maximum at time $T=2015$, by adjusting the natural growth rate of basic benefit $\lambda$ and the environmental growth rate $\psi$, we get $\lambda=0.046$ and $\psi=0.0718$ in the case of making the fitting error as small as possible. By calculating expression (23) and (24), we get:

$$
\text { Pressure index } r=0.001639572654, \quad \text { growth coefficient } \quad h=0.06869917924
$$

By the expression (19) and formulas for calculating errors, we have average growth rate of benefit and fitting error as

$$
\begin{aligned}
& \bar{g}_{1941}=\frac{h}{2006-1941} \sum_{i=` 941}^{2006} \frac{\sigma_{L}(i)}{\mu_{L}(i)}=0.01622443952 \quad \text { and } \quad \epsilon_{1941}=0.04836981174 \\
& \bar{g}_{1985}=\frac{h}{2006-1985} \sum_{i=` 941}^{2006} \frac{\sigma_{L}(i)}{\mu_{L}(i)}=0.02247541330 \quad \text { and } \quad \epsilon_{1985}=0.04125561879
\end{aligned}
$$

By expression (22) initial basic benefit and initial motivity can be respectively calculated as: $\mu_{0}=$ 0.1591428571 and $\sigma_{0}=0.005189702076$.

The solution of ARC can be calculated by (26), i.e., $L(2015)=1.328042662$, that is about 14955.76653 billions \$USD. And at the year 2015, by the expression (27), exogenous pressure will consume the benefit of $W(T)=0.1257847412$, that is about 1381.171074 billions \$USD.

Let

$$
Y(t)=L(t)=\mu_{L}(t)+0.06869917924 \sigma_{L}(t)
$$

and use the algorithm 1 in the Appendix, the ending time of ARC is $T 1=2031.929765 \approx 2032$ if US government do nothing at all from now.

The fitting curve is drawn in Fig. 4. According to the fit analysis and computation results, following control strategies are presented for referring to keep US economic growing until 2015.

Stragegy 5.1. During 1941 and 2015, the natural growth rate should be kept at $4.6 \%$ and the environmental benefit at $7.18 \%$

Under Strategy 5.1, US pressure index will be $r=0.001639572654$ and benefit growth coefficient will be $h=0.06869917924$.

Stragegy 5.2. According to US economic developing in latest 65 years, the average economic growth rate should be kept at about $1.62 \%$ during the period of 1940 and 2015 . The error of this strategy is about $4.836981174 \%$.

Stragegy 5.3. According to US economic developing in last two decades, the average economic growth rate should be kept at about $2.27 \%$ during the period of 1985 and 2015. The error of this strategy is about $4.125561879 \%$.

According to the principle of minimum error, it will be more available and effective to use strategy 5.1 and strategy 5.3 together.

\subsubsection{Arc controlling and strategies analysis for us economy to grow till $t=2025$}

If US economic benefit is controlled to reach its maximum at time $T=2025$, by adjusting the natural growth rate of basic benefit $\lambda$ and the environmental growth rate $\psi$, we get $\lambda=0.041$ and $\psi=0.062$ in the case of making the fitting error as small as possible. By calculating expression (23) and (24), we get:

$$
\text { Pressure index } \quad r=0.001639572654, \quad \text { growth coefficient } h=0.06869917924
$$

And by the expression (19) and formulas for calculating errors, we have average growth rate of benefit and fitting error as 


$$
\begin{aligned}
& \bar{g}_{1941}=\frac{h}{2006-1941} \sum_{i=`}^{2006} \frac{\sigma_{L}(i)}{\mu_{L}(i)}=0.1000304230 \quad \text { and } \quad \epsilon_{1941}=0.05107467454 \\
& \bar{g}_{1985}=\frac{h}{2006-1985} \sum_{i=` 941}^{2006} \frac{\sigma_{L}(i)}{\mu_{L}(i)}=0.1435830725 \text { and } \epsilon_{1985}=0.05935260123
\end{aligned}
$$

By expression (22) initial basic benefit and initial motivity can be respectively calculated as: $\mu_{0}=$ 0.1591428571 and $\sigma_{0}=0.005191250284$.

The solution of ARC can be calculated by (26), i.e., $L(2025)=1.689978757$, that is about 19031.71370 billions \$USD. And at the year 2025, by the expression (27), exogenous pressure will consume the benefit of $W(T)=0.8863529107$, that is about 9981.672708 billions \$USD.

Let

$$
Y(t)=L(t)=\mu_{L}(t)+0.4153822104 \sigma_{L}(t)
$$

and use the algorithm 1 in the Appendix, the ending time of ARC is $T_{1}=2042.982744 \approx 2043$ if US government do nothing at all from now. The fitting curve is drawn in Fig. 5. According to the fit analysis and computation results, we have the similar depiction to section 5.2.1.

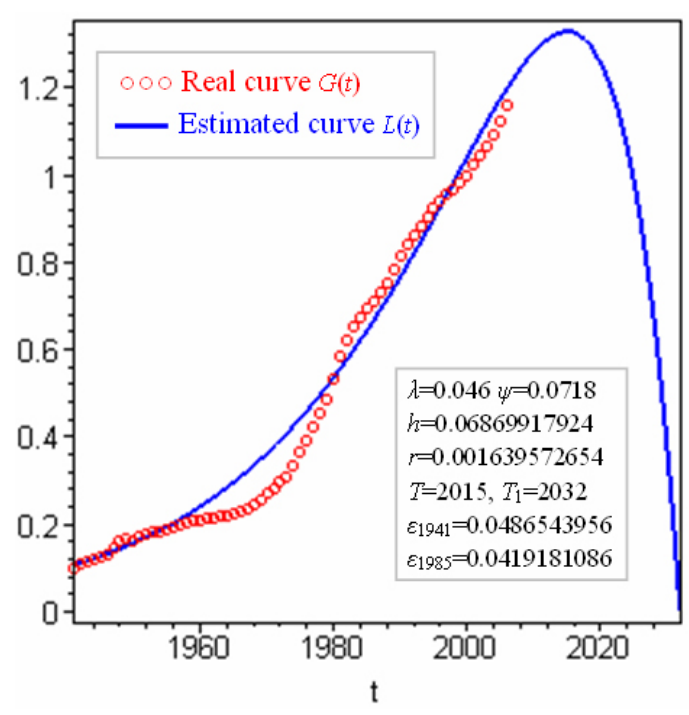

Fig. 4. Arc fitting for us gdp price index $(\mathrm{t}=2015)$

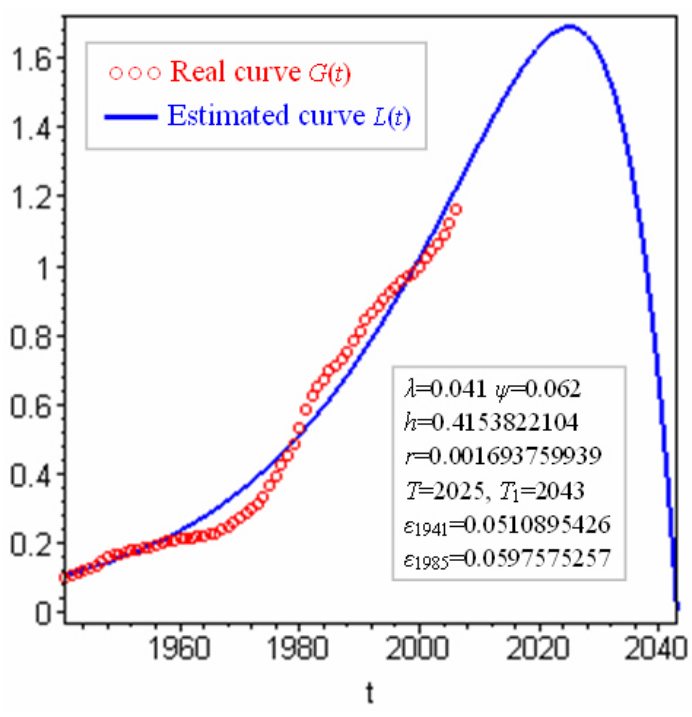

Fig. 5. Arc fitting for us gdp price index $(t=2025)$

In order to keep US economic growing until the year 2015, fitting and analysis results show that it is proper to develop economy according to the requirements of the course $L(t)=\mu_{L}(t)+0.0686991792 \sigma_{L}(t)$.

In order to keep US economic growing until the year 2025, fitting and analysis results show that it is proper to develop economy according to the requirements of the course $L(t)=\mu_{L}(t)+0.4153822104 \sigma_{L}(t)$.

\subsection{Comparisons and analytic results}

Based on the discussions above, we will make the comparisons of the two strategies for US economic growth and obtain analysis results about practical control and course characteristics.

\subsubsection{Comparative analysis}

According to Section 5.2.1 and 5.2.2, some computation data are listed in table 3, and main results of comparative analysis are following. 
Table 3. Computing data for controlling time when the maximal benefit appears

\begin{tabular}{c|c|c|c|c}
\hline $\begin{array}{c}\text { Time of maximal } \\
\text { benefit appearing }\end{array}$ & $\begin{array}{c}\text { Growth coefficient } \\
\text { and pressure index }\end{array}$ & average growth rate & $\begin{array}{c}\text { Maximal benefit } \\
\text { (billions \$USD) }\end{array}$ & $\begin{array}{c}\text { Time when the } \\
\text { benefit reaches zero }\end{array}$ \\
\hline Case1: $T=2015$ & $\begin{array}{c}h=0.06869917924 \\
r=0.001639572654\end{array}$ & $\begin{array}{c}\bar{g}_{1941}=1.62 \% \\
\bar{g}_{1985}=2.27 \%\end{array}$ & $L(2015)=14582.48506$ & $T_{1}=2031.929765$ \\
\hline Case2: $T=2025$ & $\begin{array}{c}h=0.41538221040 \\
r=0.001693759939\end{array}$ & $\begin{array}{c}\bar{g}_{1941}=9.94 \% \\
\bar{g}_{1985}=14.37 \%\end{array}$ & $L(2025)=18556.70054$ & $T_{1}=2042.982744$ \\
\hline \multirow{5}{*}{ Explanation: } & $\begin{array}{l}\text { Two fitting errors of } L(t)=\mu_{L}(t)+0.0686991792 \sigma_{L}(t) \text { are } \epsilon_{1941}=0.04836981174 \\
\text { and } \epsilon_{1985}=0.04125561879\end{array}$ \\
& $\begin{array}{l}\text { Two fitting errors of } L(t)=\mu_{L}(t)+0.4153822104 \sigma_{L}(t) \text { are } \epsilon_{1941}=0.05107467454 \\
\text { and } \epsilon_{1985}=0.05935260123\end{array}$ \\
\hline
\end{tabular}

(i) Economy can keep on growing longer if natural growth rate is properly lower. In Case 1, natural growth rate $\lambda$ is 0.046 and environmental growth rate $\psi$ is 0.0718 , which are respectively higher than $\lambda=$ 0.041 and $\psi=0.0620$ in Case 2 . This shows that although properly reducing natural growth rate will decrease the benefit growing speed in a short period of time, it can make economy growing last longer, and reduce environmental growth rate, i.e., reduce the real pressure in the economic growth. In practice, the natural growth rate can be reduced by increasing interest rate.

(ii) The smaller the pressure index is, the more efficient the ARC is. The pressure index $r=$ 0.001639572654 in Case 1 is smaller than $r=0.001693759939$ in Case 2, this means that the motivity loss in Case 1 is smaller and advance efficiency is higher under the same external environment.

(iii) Comparative analysis on real average growth rate. The real average growth rates $(\bar{g}=$ $1.62 \%$ and $\bar{g}=2.27 \%)$ in case 1 are separately lower than those $(\bar{g}=9.94 \%$ and $\bar{g}=14.37 \%)$ in Case 2. Since the growth rates required by Case 2 are higher, the economic growth by the way of Case 2 is much more difficult than that of Case 1 .

(iv) Comparative analysis on benefit wastage. According to expression (27), the benefit wastage caused by exogenous pressure in Case 1 is $W(T)=h r \kappa_{0}(1+s T)^{\theta} e^{\psi T}=0.1257847412$ (i.e., 1381.171074 billions \$USD), while that in Case 2 is $W(T)=0.8863529107$ (i.e., 9732.54 billions \$USD). Obviously, the net benefit wastage in Case 1 is much smaller than that in Case 2.

(v) Comparative analysis on error. The fitting errors of Case $1\left(\epsilon_{1} 941=0.0483698\right.$ and $\epsilon_{1} 985=$ $0.0412556)$ are separately much smaller than those of Case $2\left(\epsilon_{1} 941=0.0510747\right.$ and $\left.\epsilon_{1} 985=0.0593526\right)$. This means the Case1 is more near to the real development of US economy than Case 2. So it is easier to keep economic growth till 2015. The growth rate, however, should be controlled at about $2.266047754 \%$, and US government should impel the overall and basic economic reforms in next several years.

\subsubsection{The process of controlling economic growth based on arc}

We have the general strategic process for controlling the maximal benefit and economic growth based on the analysis above.

(i) Time controlling for maximal benefit. Controlling economic benefit to reach its maximum at a given time needs to adjust systemically such targets as pressure index $r$, growth coefficient $h$, innovational coefficient $s$, and natural growth rate $\lambda$. There are the complex relations among them, and by making use of the ARC model those relations can be described.

(ii) Controlling the growth rate. In general, environmental growth rate changes along with natural growth rate $\lambda$. Therefore, if $\lambda$ is determined, then the economic benefit can be controlled to reach maximum at a given time by computing $\psi$. On the contrary, if $\psi$ is determined, then the economic benefit can be controlled to reach maximum at a given time by adjusting $\lambda$.

(iii) Analyzing environmental pressure. When environmental growth rate, natural growth rate and the time of benefit reaching its maximum are all determined, the pressure index be computed and known, it is helpful for human to understand, analyze and eliminate the corresponding pressure factors in environment.

(iv) Enhancing net motivity. The maximal benefit can be theoretically controlled by ARC, and we needs enhance the motivity for economic developing or decrease environmental pressure to ensure that the 
real economic benefit reaches its maximum at a given time. The ways to enhance the motivity and to decrease environmental pressure includes establishing and carrying out the related economic policy, to make the effective economic reforms and innovations, and to reduce the natural growth rate properly.

\section{Conclusions and comments}

The key of ARC problem is the relationship between subject advance and object resistance. A subject which can initiatively choose and carry out strategies and an object which presents passively pressures corresponding to subject's action are always in a game. The subject needs to advance continuously to survive and develop, while the object resists the subject advancing by various environmental factors. Their relationship directly influences the subject's strategies to gain benefit. ARC is the special theory and method to research those problems.

Basing on the discussions and conclusions ${ }^{[6]}$, this paper fatherly discusses practical methods on ARC problem. The basic conclusion obtained in this paper is that enterprise management or even economic development can be analyzed and controlled in the way of ARC. In detail, the main work and basic conclusions obtained in this paper include the following four aspects.

(i) An ARC example of enterprise development is given. According to the basic characteristics of enterprise development in practice, an explanatory model of enterprise development considering environmental pressure is given. Further more, the control problem about the time at which enterprise benefit reaches its maximum is analyzed, and the control method of optimal growth is discussed. Following elementary conclusions are drawn.

- The equilibrium point of net motivity only appears in a twinkling, and net motivity is generally unbalanced.

- Marginal benefit is always decreasing after the time when net motivity reaches its equilibrium point.

- The faster the enterprise production grows, the environmental pressure the enterprise will face, at this time, the growth will keep on in a short time, the maximal benefit level (benefit equilibrium point) will arrive earlier.

- If trying to acquire the high benefits by an undue high growth may only result in lower total benefit.

(ii) A general ARC model is generalized. Based on the explanatory model of enterprise development, this paper gives a ARC model under general conditions. The main characteristic of the model is considering the environmental pressure. According to the model, following works are done.

- The general solving method for ARC is given.

- It is explained that the related result from ARC is consistent to the law of marginal income decreasing.

- The linear ARC model and its solution are discussed in detail. Furthermore, the methods to control the time at which benefit reaches its maximum on the linear ARC and to compute the optimal growth coefficient for benefit are given.

(iii) ARC theory is progressed. The ARC problem is further discussed and the existence of ARC's solution is roundly proved.

(iv) Controlling and analysis in the way of simulation is presented. Based on US GDP (chained) price index from 1940 to 2006, the ARC controlling and analysis are given. The conclusions show that:

- Economic growth will last longer if natural growth rate is suitability lower, and faster growing speed in benefit will lead to economic growth ending earlier.

- the smaller pressure index will make smaller benefit lose, so the efficiency of economic developing is higher at this time.

- the time when economic development reaches maximal level can be controlled in certain degree. Although such control relates with many factors, the controlling process can be described by using ARC model.

In addition, it is important to discuss ARC on multiple subjects and multiple phases and complex environment. And the related researches should also have the theoretical and practical value. 


\section{References}

[1] R. Barro, X. S. i Martin. Economic Growth. McGraw Hill, New York, 1995.

[2] S. Brams. Game Theory and Politics. Courier Dover Publications, 2004.

[3] A. Colman, T. Hines. Game Theory and Its Applications:In the Social and Biological Sciences. Routledge (UK), 1998.

[4] D. Croix, P. Michel. Theory of Economic Growth: Dynamics and Policy in Overlapping Generations. Cambridge University Press, 2002.

[5] F. Dai, J. Liu. The golden growth model and management strategy in macroeconomic structure. International Journal of Management Science and Engineering Management, 2007, (2): 3-22.

[6] F. Dai, J. Liu, etc. Boating against the current and the market game strategies on developing new products: Economical advance-retreat course analysis. International Journal of Management Science and Engineering Management, 2007, (2): 197-220.

[7] F. Dai, D. Zhai, etc. Boating against the current and the frame model of advance-retreat analysis for socio-economic process. Chinese Journal of Management Science, 2006, (14): 731-735.

[8] D. Fudenberg, J. Tirole. Game Theory. Massachusetts Institute of Technology, 1991.

[9] G. Gandolfo. Economic Dynamics. T Springer, 2003.

[10] R. King, C. Plosser, S. Rebelo. Growth and business cycles: Iand II. Journal of Monetary Economics, 1988, 21: 195-232.

[11] F. Kydland, E. Prescott. Time to build and aggregate fluctuations. Econometrica, 1982, 50: 1345-1370.

[12] J. Long, C. Plosser. Real business cycles. Journal of Political Economy, 1983, 91: 1345-1370.

[13] R. Lucas. Models of Business Cycles. Basil Blackwell, New York, 1987.

[14] M. Osborne. An Introduction to Game Theory. Oxford University Press, US, 2003.

[15] M. Portor. Competitive Strategy. The Free Press, 1999.

[16] R. Pouder, C. John. Geographical clusters of firms and innovation. The academy of Management Review, 2003, 21: 192-225.

[17] P. Romer. Increasing returns and long-run growth. Journal of Political Economy, 1986, 94: 1002-1037.

[18] P. Romer. Endogenous technological change. Journal of Political Economy, 1990, 98: 71-102.

Appendix (Root Solving Algorithm of Function $X(t)=0$ )

Let $t_{0}=0$, and choose the larger value of time $t_{1}>0$ for which $X\left(t_{1}\right)<0$, and give smaller real number $\epsilon>0$.

(1) Valuation: $T \longleftarrow t_{1}$

(2) Computation: $X(T)=0$

(3) If $|X(T)-0|<\epsilon$, then end. Otherwise, let $t_{1}=T$ if $X(T)<0$, and let $t_{0}=T$ if $X(T)>0$.

(4) Valuation: $T \longleftarrow \frac{t_{0}+t_{1}}{2}$, and go to (2). 